\title{
Dynamics of linear polymers in random media
}

\author{
Bikas K. Chakrabarti ${ }^{1,2}$, Amit K. Chattopadhyay ${ }^{1}$ and Amit Dutta ${ }^{1}$ \\ 1 Max-Planck-Institut für Physik komplexer Systeme, Nöthnitzer Strasse 38, 01187 Dresden, Germany \\ ${ }^{2}$ Saha Institute of Nuclear Physics, 1/AF Bidhannagar, Kolkata-70064, India
}

\begin{abstract}
We study phenomenological scaling theories of the polymer dynamics in random media, employing the existing scaling theories of polymer chains and the percolation statistics. We investigate both the Rouse and the Zimm model for Brownian dynamics and estimate the diffusion constant of the center-of-mass of the chain in such disordered media. For internal dynamics of the chain, we estimate the dynamic exponents. We propose similar scaling theory for the reptation dynamics of the chain in the framework of Flory theory for the disordered medium. The modifications in the case of correlated disordered are also discussed.
\end{abstract}

The conformational statistics of linear polymers and their dynamical properties in porous or disordered media are being investigated extensively far a long time [1-14]. The universal scaling properties of a linear polymer chain in a good solvent, which is ideally modelled in terms of the self-avoiding walk (SAW) on a regular lattice, are now well established 15.16]. However the situation is far from clear in the domain of a polymer moving in a disordered medium. Knowledge about the effects of structural disorder of the medium (or the lattice) on the polymer (or the SAW statistics) and on its dynamical properties are important both for understanding of the general influence of disorder on the critical behaviour and also for applications.

The effect of structural disorder of the lattice on the SAW statistics, in particular when disorder itself is not critical, is still under investigation. The initial indication [1] of the instability of the pure SAW fixed point in the effective $n$-vector model, was latter shown to be inappropriate [2] in the SAW $(n \rightarrow 0)$ limit. However, it seems to be established by now [5] that the proper considerations of the quenched structural disorder of the underlying lattice (fixing one or both ends of the SAW on the dilute lattice [6]) indeed induces a modified critical behaviour for the static conformational statistics of SAWs on disordered lattices. The polymer size exponent $\nu$ is defined through the relation $R_{G} \sim N^{\nu}$ connecting the radius of gyration (end-to-end distance of the SAW) $R_{G}$ of the polymer chain to the molecular weight (SAW chain length ) $N$. The disordered media are usually modelled as randomly diluted lattices with lattice site or bond concentration $p$. It is found that $\nu_{d}(\equiv \nu(p<1))$ is greater than the pure SAW size exponent $\nu(\equiv \nu(p=1))$. Of course, at the percolation threshold $p=p_{c}$, the underlying lattice becomes a fractal with effective dimensionalities less than the lattice dimension $d$. The SAW size exponent $\nu_{p_{c}}\left(\equiv \nu\left(p=p_{c}\right)\right)$ is therefore clearly different 3. 4. and in fact is larger than $\nu$ and $\nu_{d}$. The multi-fractal effects of the percolation cluster on the SAW conformational statistics at $p=p_{c}$ has also been investigated recently [11]. As mentioned already, all these investigations attempt to describe the static properties of polymers on random lattices.

Compared to the above investigations on the static properties of the polymer configurations on disordered lattices, not much is known about the effects of quenched lattice disorder on the dynamics of such linear polymers [9]. Recently, of course, the scaling theory and simulations for the reptation dynamics of polymer chains in weakly disordered lattices $(p \simeq 1)$ has been investigated [7,10]. The dynamics of un-binding of polymers in a random media has also been studied [13] and the constrained polymer dynamics has also been investigated using a variational method [14. In this report, we propose scaling theories of polymer dynamics in such porous or disordered medium using the scaling theory for polymers [15, 16] and the percolation statistics [17.

We first consider the Rouse model [15] where the interactions are local. The Brownian dynamics of the centre of mass $\vec{R}_{G}$ can be written as 16

$$
\begin{aligned}
& \frac{d R_{G}}{d t} \propto f(t) ; \\
& <f(t)>=0,<f(t) f\left(t^{\prime}\right)>=2 k_{B} T \delta\left(t-t^{\prime}\right),
\end{aligned}
$$

where $f(t)$ denotes the temperature $(T)$ dependent the random stochastic noise. One thus gets

$$
\left(\Delta R_{G}\right)^{2} \sim \frac{k_{B} T}{N} t
$$

for the average fluctuation in $R_{G}$. This gives the diffusion constant $D \equiv \Delta R_{G}^{2} / t=k_{B} T / N$. In the nodelink-blob model [17] describing the percolation cluster, the occupied sites or bonds form a super-lattice with lattice constant $\xi_{p} \sim\left(p-p_{c}\right)^{-\nu_{p}}$, where $\xi_{p}$ is the percolation correlation length. In this model, however another length-scale, namely "chemical length" (along the lattice edge) scaling as $l_{p} \sim\left(p-p_{c}\right)^{-\zeta_{p}}$, appears due to the tortocity of the percolating paths. Moreover, there exists random blobs (multiply connected regions) and dangling ends. Assuming the possibility of Brownian diffusion on such super-lattices, we find that the diffusion constant $D$ of a Gaussian chain can be written as (cf. [15, 16]).

$$
\begin{aligned}
D & \sim \frac{\frac{\left(\Delta R_{G}\right)^{2}}{\xi_{p}^{2}}}{t} \sim\left(p-p_{c}\right)^{2 \nu_{p}}\left(\frac{\left(\Delta R_{G}\right)^{2}}{t}\right) \\
& \sim\left(p-p_{c}\right)^{\alpha_{R}}\left(\frac{k_{B} T}{N}\right)
\end{aligned}
$$


where

$$
\alpha_{R}=2 \nu_{p}
$$

Here the radius of gyration $R_{G}$ is scaled appropriately on the percolation cluster (super-lattice at $p>p_{c}$ ) by $\xi_{p}: R_{G} \rightarrow R_{G} / \xi_{p}$. It may be noted here that the chainlength $N$ appears here in the above diffusion equation as mass and thus remains unchanged and should not be scaled with the chemical length $l_{p}$ (see however the later discussion on the reptation dynamics). Clearly, the exponent $\alpha_{R}$ is always positive and therefore $D$ decreases to zero here as $p \rightarrow p_{c}$.

If one considers the long-ranged (hydrodynamic) interactions on top of the Rouse-like interaction, as in the Zimm-model, one gets (cf. 150)

$$
\begin{aligned}
D & \sim \frac{\frac{\left(\Delta R_{G}\right)^{2}}{\xi_{p}^{2}}}{t} \sim\left(p-p_{c}\right)^{2 \nu_{p}}\left(\frac{\left(\Delta R_{G}\right)^{2}}{t}\right) \\
& \sim\left(p-p_{c}\right)^{2 \nu_{p}}\left(\frac{k_{B} T}{N}\right) \int d r r^{(d-1)} g(r) \mu(r) \\
& \sim\left(p-p_{c}\right)^{2 \nu_{p}}\left(\frac{k_{B} T}{\left(R_{G} / \xi_{p}\right)}\right) \sim\left(p-p_{c}\right)^{\alpha_{Z}}\left(\frac{k_{B} T}{N^{\nu_{d}}}\right),
\end{aligned}
$$

using similar scaling of the appropriate variables. Here $g(r) \sim\left[N / R_{G}{ }^{d}\right] g\left(r / R_{G}\right)$ denotes the pair correlation function with $R_{G}$ scaled by $\xi_{p}$ and $\mu(r) \sim r^{-1}$ denotes the mobility function. We therefore get

$$
\alpha_{Z}=\nu_{p} .
$$

Here also the centre of mass diffusion constant $D$ decreases to zero following the above power law, as $p \rightarrow p_{c}$.

For the internal dynamics or local excitations of the polymer chain, one can estimate the dynamic exponent $z$ following the same scaling argument [16] for the pure case. Writing the dynamical structure factor as

$$
\begin{aligned}
g(k, t) & =N g\left(k\left(\frac{R_{G}}{\xi_{p}}\right), t \frac{D}{\left(R_{G} / \xi_{p}\right)^{2}}\right) \\
& \sim N k\left(\frac{R_{G}}{\xi_{p}}\right)^{-\frac{1}{\nu_{d}}} \tilde{g}\left(t D\left(\frac{R_{G}}{\xi_{p}}\right)^{-2}\left[k\left(\frac{R_{G}}{\xi_{p}}\right)\right]^{z}\right) \\
& \sim \tilde{g}\left(t k^{z} D R_{G}{ }^{z-2} \xi_{p}^{2-z}\right) \\
& \sim \tilde{g}\left(\left(p-p_{c}\right)^{\gamma} k^{z} t\right) .
\end{aligned}
$$

Since the local excitations (for $k R_{G} \gg 1$ ) are independent of $N$, assuming $D \sim\left(p-p_{c}\right)^{\alpha} N^{-\beta}$ (with $\alpha=$ $2 \nu_{p}, \beta=1$ for the Rouse model (Eq. (3)) and $\alpha=\nu_{p}$, $\beta=\nu_{d}$ (Eq. (6)) for the Zimm model, one gets

$$
z=2+\frac{\beta}{\nu_{d}}, \text { and } \gamma=\alpha+\nu_{p}(z-2) .
$$

Hence $z=3$ for all dimensions in the Zimm model, while $z$ changes to $2+1 / \nu_{d}$ for the disordered case in the Rouse model and its magnitude becomes equal to 4 for $d \geq 6$.
We have assumed in the above discussion, the possibilities of the Rouse or Zimm dynamics of the polymer chain in good solvents within the pores of the random media. This obviously necessitates the correlated percolation picture of the porous media, and hence the appropriate modifications [12 of the polymer statistics in such medium (see below). However, very near the percolation threshold, the polymer dynamics can perhaps be only reptation type [15] along the constrained pore tubes. Following the earlier investigations [7.10], the diffusion constant $D$ for the polymer, making reptation dynamics in the disordered media, can be written as

$$
\begin{aligned}
D & \sim\left(\frac{\left(\frac{R_{G}}{\xi_{p}}\right)^{2}}{\tau}\right) \exp \left(-\frac{\Delta F\left(R_{G}\right)}{k_{B} T}\right) \\
& \sim \frac{\left(p-p_{c}\right)^{2 \nu_{p}}}{N^{3-2 \nu_{d}}} \exp \left(-\frac{\Delta F\left(R_{G}\right)}{k_{B} T}\right),
\end{aligned}
$$

where $R_{G} \sim N^{\nu_{d}}, \tau \sim N^{3}$ and $\Delta F\left(R_{G}\right)$ denotes the fluctuation in the polymer free energy due to the structural disorder of the medium. A simple Flory type estimate for the free energy $F$ can be written, following Smailer et al [7], as

$$
F \sim\left(\frac{\frac{R}{\xi_{p}}}{\left(\frac{N}{l_{p}}\right)^{\nu}}\right)^{1 /(1-\nu)}+\left(\frac{\left(\frac{N}{l_{p}}\right)^{2}}{\left(\frac{R}{\xi_{p}}\right)^{d}}\right)^{1 / 2},
$$

where $\nu(=3 /(d+2))$ is the SAW size exponent on the pure lattice and the second term, arising due to the random fluctuation of the density-density (excluded volume) interaction, gives the configurational fluctuation $\Delta F$. The most important point here is the rescaling of the polymer mass with the chemical length $l_{p}$. Until the above equation, we considered scaling of only the fluctuation $\Delta R_{G}$ by the lattice constant $\xi_{p}$ of the percolating super-lattice, and not of the polymer mass $N$. In the above expression for the Flory free energy, on the other hand, one has to scale as well the chain length or mass $N$ by $l_{p}[8]$. This is necessary here to cast the free energy in the disorderd media in the pure lattice form; or in other words, as in the free energy, both temperature and mass renormalisatins are allowed. The minimisation, with respect to $R$ of the above free-energy gives the polymer size as

$$
\begin{aligned}
R_{G} & \sim\left(p-p_{c}\right)^{-\delta} N^{\nu_{d}} ; \\
\nu_{d} & =\frac{2}{2+d(1-\nu)}, \quad \delta=\nu_{p}-\zeta_{p} \nu_{d} .
\end{aligned}
$$

The above result may also be obtained by scaling the relation $\left(R_{G} \sim N^{\nu_{d}}\right)$ connecting the radius of gyration to the polymer chain length: $R_{G} \rightarrow R_{G} / \xi_{p}$ and $N \rightarrow N / l_{p}$ [18]. The value of the exponent $\delta$ is positive [17] for $d=2$ and 3 . This indicates that the polymer swells (for fixed $N)$ as $p \rightarrow p_{c}$. The possible negative value of $\delta$ for $d \geq 4$ may indicate localisation of the polymer in higherdimnensions (Gaussian chains) in the random media [9]. 
The fluctuation $\Delta F$ can therefore be written as

$\Delta F \sim\left(p-p_{c}\right)^{-\phi} N^{\chi} ; \quad \phi=\frac{d}{2} \nu_{p}-\zeta_{p}, \quad \chi=\frac{\nu_{d}}{2}(2-d \nu)$.

It may be noted from the above expression that $\nu_{d}>\nu$ for all $d<4$ and we assume $\nu_{d}=\nu=1 / 2$ for $d \geq 4$. The values of the exponents $\phi$ and $\chi$ are positive 17] for $d<4$. The above Eqs. (10) and (12) together describes the scaling behaviour of the diffusion constant $(D)$ of the reptation motion for the polymer chain in the disordered medium. We find that for $d<4, D$ decreases exponentially not only as the chain length $N$ increases but also as $p \rightarrow p_{c}$. At $d=4, \chi$ vanishes while $\phi$ becomes slightly negative so that the diffusion constant $D$ in (12) becomes independent of $N$ and decreses with $p$ approaching $p_{c}$. This seems to be related to the possible localisation of polymers in higher-dimensions as discussed already.

As mentioned already, these dynamical properties of polymers in porous media demand the consideration of the correlated randomness of the percolating cluster. Very recently, Blavasts'ka et al [12 have studied the static scaling properties of polymers on a $d$-dimensional random lattice with "correlated" randomness that has a power-law fall of the form $1 / r^{a}$ for large separation $r$ [19]. Similar Flory-type estimate for the free energy $F$ in the case of relevant long-range correlation $(a<d)$ will be given by Eq. (10) where the spatial dimensionality $d$ in the second term will be replaced by the parameter $a$ denoting the range of interaction. For $a \geq 4$, this fluctuation term becomes irrelevant and thus we get the mean field critical behaviour. Using therefore the expression (11) for the Flory estimate of $\nu_{d}$ for the non-trivial cases $a<4(d \simeq 4)$, we get

$$
\nu_{d}=\frac{2}{2+a\left(\frac{d-1}{d+2}\right)} \simeq \frac{1}{2}+\frac{\delta}{16},
$$

where $\delta=4-a$ and $\nu=1 / 2+(4-d) / 12$. Clearly, this size exponent $\nu_{d}$, obtained via Flory theory, does not match the renormalisation group result [12] even in the first-order in $\delta$. This is is similar to the failure of the Flory approximation for the exponent $\nu$ in the first-order in $(4-d)$ even in the case of pure lattice (with short-range interactions) [15]. It may however be noted that this result for $\nu_{d}$ is for the long-range case $a<d$, where the renormalisation group study in 12] failed to locate any stable fixed point. For $a>d$, the above method clearly yield the pure (short-range) SAW result. However, the renormalisation group study suggests the existence of a long-range fixed point in this region [12].

In conclusion, we have studied the dynamical properties of lattice models of a linear polymer on a percolating lattice model of the random or porous media, using simple scaling ideas for both the polymer chain and the disordered media. The results for the dynamical exponent $z$ describing the internal dynamics, and the diffusion coefficient $D$ of the center of mass of the polymer chain, using both the Rouse and the Zimm model, have been investigated for such disordered media. We find that while the diffusion coefficient decreases with $p-p_{c}$, following power laws in both the cases, the dynamic exponent $z$ remains unchanged to its pure value 3 for the Zimm model but gets modified for the Rouse model. Applications of the Flory theory for the polymer free-energy and the scaling theory of the percolation disorder indicate that the reptation diffusion coefficient $D$ of the polymer chain decreases exponentially with the chain length $(N)$ and also inversely with the deviation $\left(p-p_{c}\right)$ from the percolation threshold $p_{c}: D \sim \exp \left[-N^{\chi}\left(p-p_{c}\right)^{\phi}\right]$, where the exponents $\chi$ and $\phi$ are both positive and related to the polymer size and the percolation exponents respectively. Thus, based on a simple Flory-type theory, we have extended a few dynamical properties of linear polymers near the percolation threshold and have also discussed the case of correlated randomness.

\section{ACKNOWLEDGEMENT}

BKC is grateful Peter Fulde for his kind hospitality at MPIPKS, Dresden, Germany.

[1] B. K. Chakrabarti and J. Kertesz, Z. Phys. B 44, 221 (1981).

[2] A. B. Harris, Z. Phys. B 49, 347 (1983).

[3] S. B. Lee and H. Nakanishi, Phys. Rev. Lett. 61, 2022 (1988).

[4] Y. Meir and A. B. Harris, Phys. Rev. Lett. 63, 2819 (1989).

[5] C. Vanderzande and A. Kamoda, Phys. Rev. A 45, R5335 (1992).

[6] P. Grassberger, J. Phys. A 26, 1023 (1993).

[7] I. Smailer, J. Machta and S. Redner, Phys. Rev. E 47, 262 (1993).

[8] K. Barat and B. K. Chakrabarti, Phys. Rep. 258, 377 (1995).

[9] A. Baumgartner and M. Muthukumar, Adv. Chem. Phys. 94, 625 (1996).

[10] D. Cule and T. Hwa, Phys. Rev. Lett. 80, 3145 (1998).

[11] A. Ordemann, M. Porto, H. E. Roman, S. Havlin and A. Bunde, Phys. Rev. E 616858 (2000); A. Ordemann, M. Porto, H. E. Roman and S. Havlin, Phys. Rev. E 63, 020104 (R) (2001).

[12] V. Blavats'ka, C. von Ferber and Yu. Holovatch, Phys. Rev. E 64, 041102 (2001).

[13] S. M. Bhattacharjee and A. Baumgartner, J. Chem. Phys. 107, 7571 (1997).

[14] M. Koch, J-U. Sommer and A. Blumen, J. Phys. A 30, 5007 (2001). 
[15] P. G. de Gennes, Scaling Concepts in Polymer Physics, Cornell Univ. Press, Ithaca (1979).

[16] M. Doi and S. F. Edwards, The theory of Polymer Dynamics, Clarendon Press, Oxford (1986).

[17] D. Stauffer and A. Aharony, Introduction to Percolation
To Theory, Taylor and Francis, London (1992)

[18] A. K. Roy and B. K. Chakrabarti, J. Phys. A, 20, 215 (1987).

[19] A. Weinrib and B. I. Halperin, Phys. Rev. B 27, 413 (1983). 